EPJ Web of Conferences 87,02009 (2015)

DOI: $10.1051 /$ epjconf/ 20158702009

(C) Owned by the authors, published by EDP Sciences, 2015

\title{
Performance History and Upgrades for the DIII-D Gyrotron Complex
}

\author{
J. Lohr ${ }^{1, a}$, J.P. Anderson ${ }^{1}$, M. Cengher ${ }^{1}$, R.A. Ellis ${ }^{2}$, Y.A. Gorelov ${ }^{1}$, E. Kolemen ${ }^{2}$, T. Lambot ${ }^{3}$, D.D. Murakami ${ }^{4}$, \\ L. Myrabo ${ }^{5}$, S. Noraky ${ }^{1}$, K.L. Parkin ${ }^{3}$, D. Ponce ${ }^{1}$, and A. Torrezan ${ }^{1}$ \\ ${ }^{1}$ General Atomics, P.O. Box 85608, San Diego, California 92186-5608, USA \\ ${ }^{2}$ Princeton Plasma Physics Laboratory, P.O. Box 451, Princeton, New Jersey 08543-0451, USA \\ ${ }^{3}$ Carnegie Mellon University, Moffett Field, California 94035, USA \\ ${ }^{4}$ Experimental Aero-Physics Branch, NASA Ames Research Center, Moffett Field 94035, California, USA \\ ${ }^{5}$ Lightcraft Technologies Inc., 1914 Walloomsac Rd, Bennington, Vermont 05201, USA
}

\begin{abstract}
The gyrotron installation on the DIII-D tokamak has been in operation at the second harmonic of the electron cyclotron resonance since the mid-1990s. Prior to that a large installation of ten $60 \mathrm{GHz}$ tubes was operated at the fundamental resonance. The system has been upgraded regularly and is an everyday tool for experiments on DIII-D.
\end{abstract}

\section{Introduction}

The gyrotron system on the DIII-D tokamak [1] presently comprises six operating tubes, with infrastructure for eight, all of which nominally generate $1 \mathrm{MW}$ for short pulses and about $800 \mathrm{~kW}$ for operational pulse lengths, which are limited administratively to $5 \mathrm{~s}$, consistent with the DIII-D pulse length. The measured transmission line efficiencies are $\sim 75 \%$, in agreement with expectations for the 90-100 m line lengths and numbers of miter bends. All of the gyrotrons operate at $110 \mathrm{GHz}$. Two are a depressed collector design, part of a recent upgrade, while the others are older undepressed diode tubes [2]. The installation is transitioning, as funding permits, to a new series of gyrotrons with a higher frequency of 117.5 GHz, the first example of which has demonstrated $1.8 \mathrm{MW} 5 \mathrm{~ms}$ pulse operation at $60 \mathrm{~A}$ and $1.5 \mathrm{MW}$ at $50 \mathrm{~A}$ in initial testing at CPI [3]. Two gyrotrons are presently undergoing repair and will be returned to service. One developed an internal water leak and a second failed due to a mechanical problem with the electron gun. Both of these failed tubes had been in service for more than 5 years. Maximum injected $\mathrm{rf}$ power has been about 3.5 MW from the whole system and this performance level is expected to continue to be realized during the 2014 experimental campaign. The gyrotron performance histories are summarized in Fig. 1 along with operational injected power values for the 2013 campaign.

The gyrotron system on DIII-D began operation with 1 MW class tubes in 1997 and since then has been steadily increased in capability [4]. The overall reliability over the past 9 years on a single gyrotron basis has been

\footnotetext{
a Corresponding author: lohr@fusion.gat.com
}

about $85 \%$ even though four of the gyrotrons are operated in pairs on single power supplies, hence a fault in one causes two tubes to be lost for the plasma shot. An example of the reliability accounting algorithm is: If there are 5 gyrotrons up and running and all are asked to fire $5 \mathrm{~s}$ pulses; but 4 fire for the requested pulse length and one faults after $3 \mathrm{~s}$, this would be an $80 \%$ success for that one plasma shot. There are a disproportionate number of faults early in the experimental day before operation is fully stabilized.

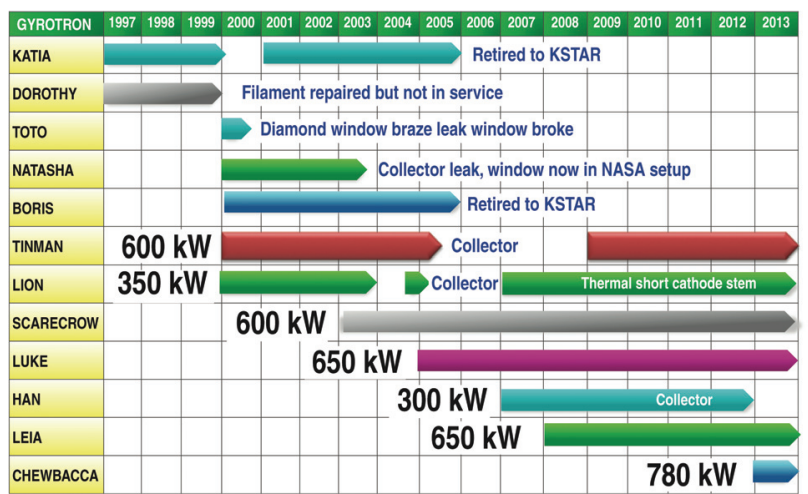

Figure 1. The history of $1 \mathrm{MW}$ class gyrotrons on DIII-D is summarized since 1997 when the first Gycom $110 \mathrm{GHz}$ tubes were installed. There have been failures and repairs, but the system has been injecting $\sim 3 \mathrm{MW}$ or more for more than 5 years.

In addition to the more familiar plasma physics and fusion related experiments, the electron cyclotron heating $(\mathrm{ECH})$ system is also being used in an experiment in conjunction with NASA as the power source for small 
rockets being accelerated by microwave heating of fuel without use of an oxidizer [5]. Tests of the design of suitable heat exchangers and engines are ongoing.

The long term upgrade plan for the DIII-D gyrotron complex calls for 10 gyrotrons with a regular replacement program leading to most or all of the tubes generating at 117.5 GHz with nominal output power of 1.5 MW per unit. This will require upgrades to the building, new high voltage power supplies, one additional dual launcher, and controls to expand beyond the present 8 gyrotron system.

\section{Launcher performance upgrade}

Rapid motion of the launcher steering mirrors, particularly in the poloidal, or vertical, plane can greatly enhance the utility of the ECH system. On DIII-D, the electron cyclotron second harmonic resonance cuts across nearly all flux surfaces for most plasma equilibria. Therefore, being able to change the point where the resonance, the rf beam trajectory, and the desired target flux surface intersect can permit localized heating and current drive (ECCD) to be applied as required in an evolving discharge. In many discharges, where neoclassical tearing modes (NTMs) or sawtooth oscillations $^{5}$ have grown and confinement is being adversely affected, being able to place the ECCD at the appropriate flux surface can restore the nested flux geometry or limit growth of the instability and save the shot.

There are 4 dual ECH launcher assemblies on DIII-D, each of which can handle full power from two of the gyrotrons, with individual waveguides and fully articulating steering mirror assemblies. Using these steering mirrors, the $\mathrm{rf}$ beams can be directed over a $40^{\circ}$ range in the vertical, poloidal, plane and $\pm 20^{\circ}$ horizontally, which accesses the plasma center and above, while providing current drive in both the co- and counter-directions. This steering capability is being upgraded with higher speed drive motors and new position readback encoders, which have demonstrated a full $40^{\circ}$ poloidal scan in $<200 \mathrm{~ms}$ and about $2 \mathrm{~mm}$ positioning accuracy for the $\mathrm{rf}$ beam at the plasma center in the initial tests. The aiming and appropriate polarizer angles can be set up and fixed prior to a plasma shot with preprogrammed time dependence of the rf power, but aiming and output power control can also be assumed by the DIII-D Plasma Control System (PCS). Under PCS control, realtime equilibrium calculations or preprogrammed scans guide the aiming. Among the inputs, all of which are calculated in realtime during the plasma shot, are EFIT equilibria normalized by motional Stark effect (MSE) measurements to provide the q surface calculation, mode detection using magnetic fluctuation data and the analysis code NEWSPEC, Thomson scattering $\mathrm{n}_{\mathrm{e}}(\mathrm{r})$ and $\mathrm{T}_{\mathrm{e}}(\mathrm{r})$ profiles, and fast ray tracing using TORBEAM (checked by comparison with TORAY). These diagnostic inputs, provide the basis for determining the amount and location of required heating or current drive. The result is the ability to fully suppress MHD instabilities [6] such as NTMs, to influence the j(r) and $\mathrm{T}_{\mathrm{e}}(\mathrm{r})$ profiles and to study transport and other fundamental aspects of the discharge. The ability to track a desired flux surface in realtime using TORBEAM for the $\mathrm{rf}$ beam trajectory is demonstrated in Fig. 2. One major advantage of rapid poloidal scanning is that a single frequency system can be used for several tasks. For example, the poloidal scan on the DIII-D system can transition between sawtooth relevant aiming and NTM suppression in $\approx 50 \mathrm{~ms}$, a time short compared with the growth time of the NTM, as suggested in Fig. 2, and rapid enough to keep incipient sawtooth amplitudes small.
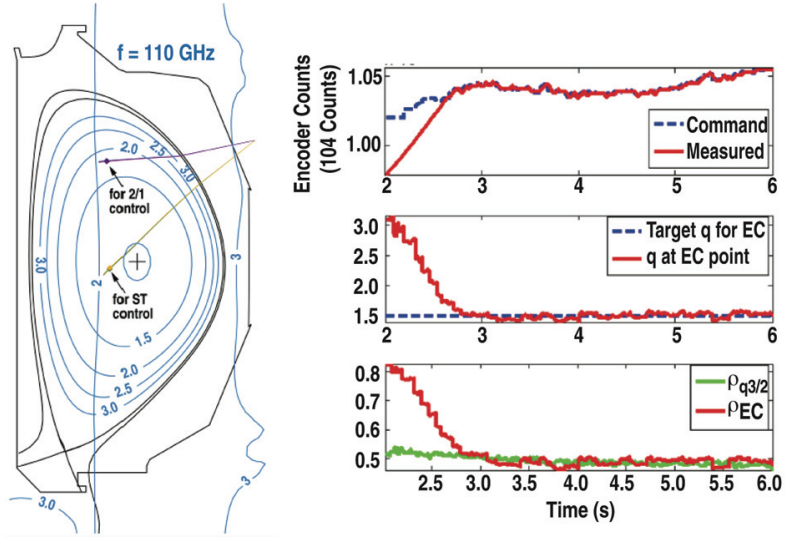

Figure 2. Tracking of the $q=3 / 2$ flux surface as calculated by both TORAY and TORBEAM agrees well with the location of the surface from MSE EFITS. The poloidal aiming of the launcher mirrors can quickly move to and follow the location of this surface.

It is common that a sequence of NTMs, such as the $\mathrm{m} / \mathrm{n}=3 / 2$ then $\mathrm{m} / \mathrm{n}=2 / 1$ modes, is observed, with the $2 / 1$ mode leading to a disruption of the plasma. This will be particularly dangerous in the ITER device. Suppression of either the $3 / 2$ or the $2 / 1$ mode can interrupt the progression to a disruptive termination, but this requires accurate positioning of the ECCD. When the ECCD location is well-aligned with that required to restore the current inside the magnetic island, NTMs can be suppressed with only modest EC power, as seen, for example, in Fig. 3, where the poloidal aiming was preset to drive current in excess of the bootstrap current on the $\mathrm{q}=2$ flux surface where the $\mathrm{m} / \mathrm{n}=2 / 1 \mathrm{NTM}$ is localized.

To provide the high speed aiming, dc electric motors developing $119 \mathrm{mN}-\mathrm{m}$ of torque at 5,500 rpm were installed and the earlier mechanical position encoders were replaced with 16 bit magnetic encoders capable of the rapid readout speeds required. These components were tested over the period of a year for neutron immunity and it later was found that the DIII-D environment also required magnetic shielding to reduce the ambient field by about a factor of two. The mirrors can be controlled and moved either by a single command from the ECH system or by a series of motion commands generated by the PCS. At present, only the poloidal motion can be commanded in real time by the PCS. The communications protocol is UDP, which puts the responsibility for error checking on the application. The encoder read time is about $700 \mu$ s and the overall communications latency is about $1 \mathrm{~ms}$. The PCS polls the system every $2 \mathrm{~ms}$. Using the highest motor speed, which 
is determined by a combination of pulse duty cycle and Voltage, the full $40^{\circ}$ poloidal scan range of the mirrors requires just over $100 \mathrm{~ms}$. A set of port box limits, determined by infrared imaging of the rf beam inside the DIII-D vacuum vessel, is applied in software, which causes slowing of the motion when the limits are approached. Microswitches remove motor power when the drive mechanism is moved too close to the hard stop limits defined by the drive mechanism itself.
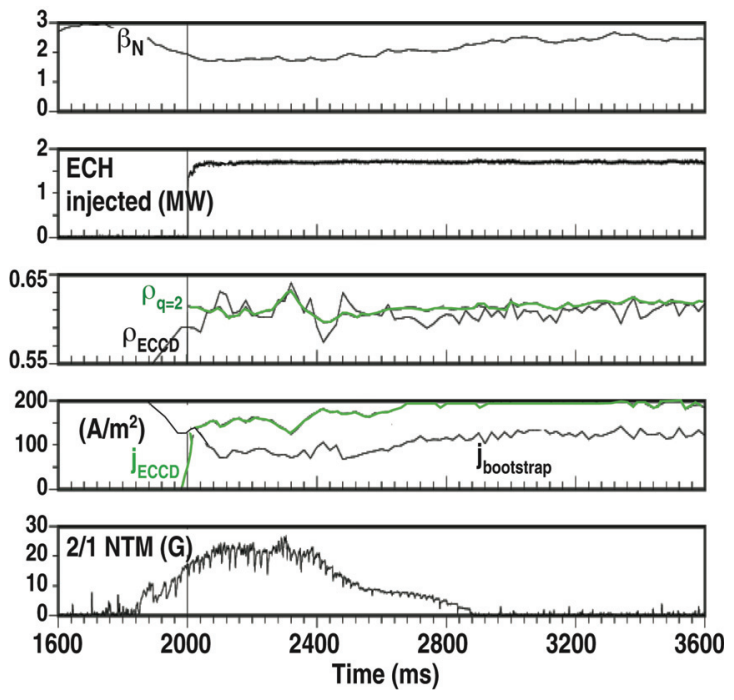

Figure 3. Suppression of an $\mathrm{m} / \mathrm{n}=2 / 1 \mathrm{NTM}$ using $<2.0 \mathrm{MW}$ ECCD with good pre-set alignment of the bootstrap and ECCD currents. The discharge recovered the original $\beta_{\mathrm{N}}$ once the NTM was suppressed.

\section{$3 \mathrm{ECH}$ thrust generation}

A novel proposal for ground-based heating of fuel in small rocket engines without requiring oxidizer to support combustion [5] is being explored using one of the DIII-D gyrotrons. The tests are being performed in a large mobile laboratory based on a heavy truck trailer platform. The microwaves from the gyrotron are switched from the normal paths, to a path leading through a hole in the wall of the DIII-D gyrotron vault and through the wall of the trailer parked outside. The trailer contains an optical table, various focus mirrors, stray rf absorbers and diagnostic cameras to observe the heating of the motors by the microwave beam. The propellant gasses are in high pressure bottles stored outside the trailer. Full implementation of the concept will require a significant infrastructure investment.

The microwaves start out at the waveguide switch in $31.75 \mathrm{~mm}$ diameter evacuated corrugated circular waveguide. In the space between the laboratory wall and the trailer, the guide uptapers to $63.5 \mathrm{~mm}$ diameter circular corrugated waveguide, passes two miter bends and arrives at the optical table, where it is launched into free space toward the focus mirrors and the targets. A boron nitride resonant window from a retired gyrotron is mounted near the end of the waveguide, protected by arc detectors. A diamond isolator window, previously mounted in the approximate middle of the waveguide run was broken when an rf-driven arc formed in the atmospheric pressure portion of the waveguide line and the pressure wave, calculated at about 3 atm, traveled back to strike the window. Placing the $\mathrm{BN}$ window at the end of the waveguide run will prevent such a pressure wave from forming. Thus far the experiments have demonstrated the transport system can route high power microwaves into the trailer to characterize the beam and heat highly absorptive coated alumina tubes, which are being considered for one type of thruster. The setup is shown in Fig. 4, with the infrared image of the beam and heating of the tubes.

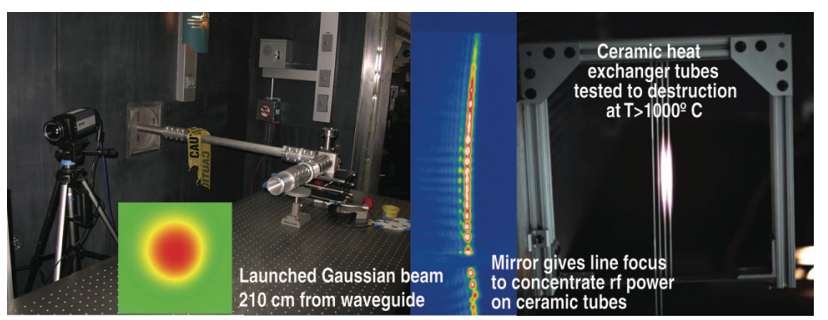

Figure 4. The setup for the microwave rocket experiments includes a waveguide connection to one of the DIII-D gyrotrons and an experimental laboratory in a large truck trailer. In the initial experiments heating of the ceramic heat exchanger tubes was demonstrated.

\section{Upgrades and plans}

The next group of gyrotrons to be added to the DIII-D complex will all be depressed collector tubes [4]. Two of these are $110 \mathrm{GHz}$ tubes and all the rest will generate 117.5 GHz, a frequency which is better for current drive and heating at the highest performance levels on DIII-D. The two $110 \mathrm{GHz}$ tubes are both installed and the first of these has been in operation for one experimental campaign. The second is in the early stages of conditioning to full power. The free space tests of the rf beam quality for this tube showed excellent Gaussian beam quality and mode stability, as seen in Fig. 5. Although the $110 \mathrm{GHz}$ tubes are designed to generate 1.2 MW, this power level has only been achieved for short pulses on the tube currently in regular service. Cooling of the cathode during long pulses, for which the usual filament boost program could not compensate, has reduced the output to approximately $950 \mathrm{~kW}$ on this gyrotron. The $110 \mathrm{GHz}$ gyrotrons operate in the $\mathrm{TE}_{22,6}$ mode, the same mode as the older diode tubes, however the $117.5 \mathrm{GHz}$ gyrotrons will operate in the $\mathrm{TE}_{20,9}$ mode, which has been shown on the first of the new gyrotrons to provide good mode separation and stable operation for short pulse power output of $1.8 \mathrm{MW}$.

Although the new $110 \mathrm{GHz}$ tubes are being placed in service, there have been some problems. The first tube was found to have a number of collector cooling passages blocked by braze material, which reduced the predicted lifetime from greater than 100,000 pulses to about 30,000 pulses, still an acceptable figure for the DIII-D installation. The second of these tubes was difficult to operate during factory testing and there was evidence of arcing on the outside of the cathode insulator. This tube is currently being tested at DIII-D. The first of the 


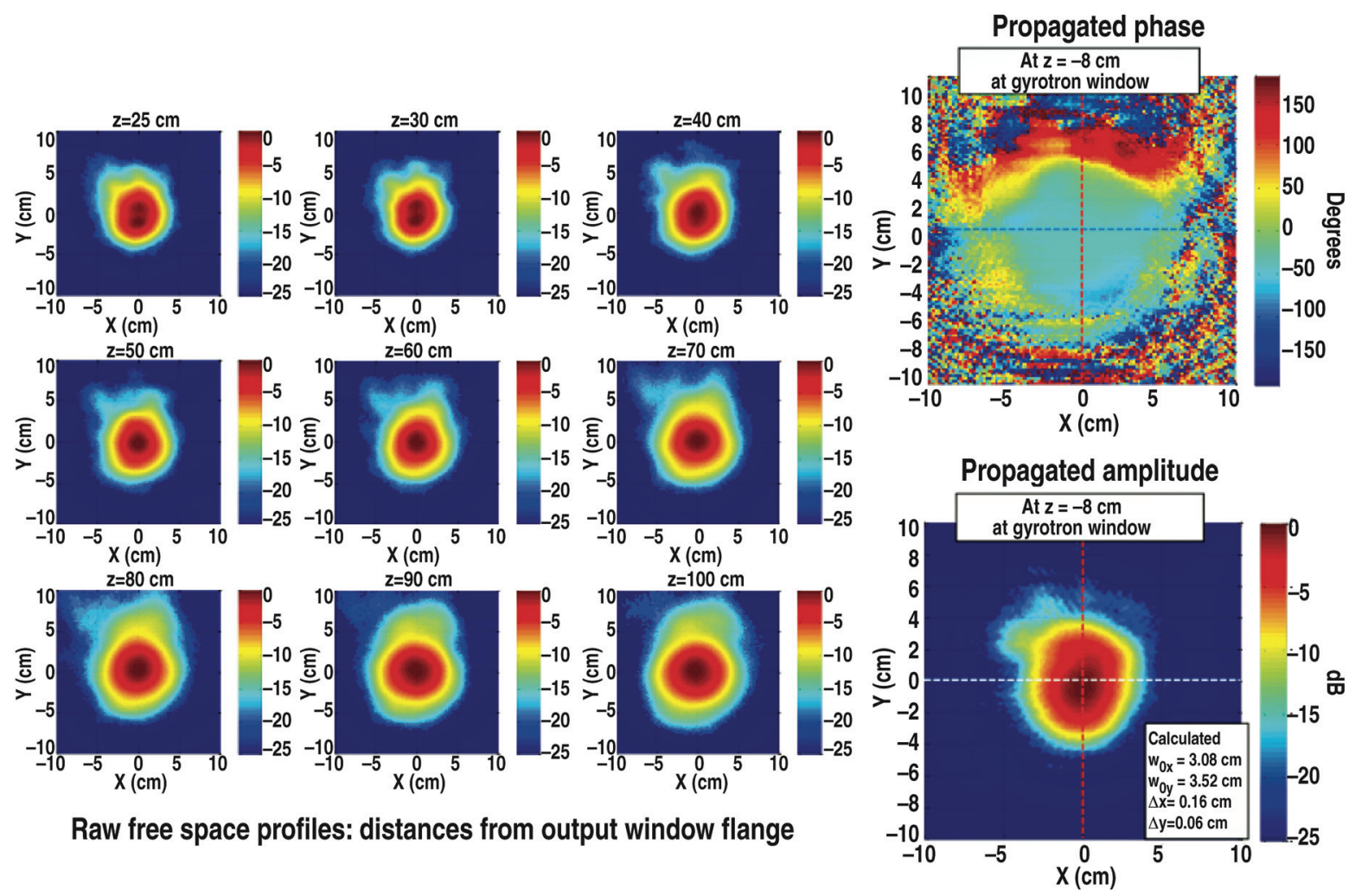

Figure 5. The nine free space amplitude profiles on the left were measured using an infrared camera beginning at the gyrotron output flange and going out $100 \mathrm{~cm}$ in $10 \mathrm{~cm}$ increments. The phase retrieval analysis was used to propagate the beam amplitude and phase profiles back to the location of the output window, at which point the Gaussian content was $93.2 \%$. There is a small vertical distortion in the raw data due to convection.

117.5 GHz gyrotrons, despite its excellent short pulse power performance, was not able to stand off the designed total voltage of $105 \mathrm{kV}$ when the magnetic field was on. This tube is planned for rework and additional testing. For all these gyrotrons, however, the rf beam quality is excellent and the coupling to the waveguide for the operational tube is the best among the gyrotrons presently in service. The experience with these tubes suggests that the goal of 1.5 MW gyrotrons for ITER is within the realm of possibility.

\section{Summary}

The gyrotron complex on DIII-D is operating routinely with good reliability and injected power between 3.0 and 3.5 MW for the most recent experimental series. In addition to tokamak physics experiments, microwave driven thruster tests have been performed. The upgrade path includes a total of 10 gyrotrons, 5 dual launchers and a change in operating frequency from $110 \mathrm{GHz}$ to $117.5 \mathrm{GHz}$.

\section{Acknowledgment}

This work was supported by the US Department of Energy under DE-FC02-04ER54698 and DE-AC0209CH11466.

\section{References}

1. J. Lohr, et al., Fusion Sci. Technol. 48, 1226 (2005)

2. G.S. Nusinovich, et al., J. Infrared, Millimeter, and Terahertz Waves 35, 325 (2014).

3. Communications and Power Industries, Palo Alto, California, USA

4. M. Cengher, et al., IEEE Transactions on Plasma Science 2014, DOI : 10.1109/TPS.2013.2292299

5. Kevin L.G. Parkin, "The Microwave Thermal Thruster and Its Application to the Launch Problem," Ph.D. Thesis, California Institute of Technology (2006)

6. John Wesson, Tokamaks, $4^{\text {th }}$ Ed, Oxford University Press (2011) 